\title{
Ultrahigh-channel-count fiber Bragg grating based on the triple-sampling method
}

\author{
Xuxing Chen, Junya Hayashi, and Hongpu Li* \\ Department of Electrical and Electronic Engineering, Shizuoka University, Johoku 3-5-1, \\ Hamamatsu 432-8561, Japan \\ *Corresponding author:dhli@ipc.shizuoka.ac.jp
}

\begin{abstract}
A triple sampling method enabling to have excellent channel uniformity and high in-band energy efficiency is firstly proposed for the design of an ultrahigh-channel-count fiber Bragg grating (FBG), which is based on the simultaneous utilization of two amplitude-assisted phase sampling (AAPS) functions and a phase-only sampling (POS) function. As an example, one linearly chirped FBG with consecutive 1215 channels enabling to cover all fiber telecom bands $(\mathrm{O}+\mathrm{E}+\mathrm{S}+\mathrm{C}+\mathrm{L}+\mathrm{U})$ is numerically demonstrated, which has a length of $9 \mathrm{~cm}$, a dispersion of $-1360 \mathrm{ps} / \mathrm{nm}$, and a channel spacing of $50 \mathrm{GHz}$. The maximum index-change required for 10 $\mathrm{dB}$ strength of the FBG is less than $6 \times 10^{-3}$.
\end{abstract}

(C) 2010 Published by Elsevier B.V.

Keywords: Fiber Bragg grating; Wavelength filtering devices; Fiber optical components; Dispersion. 


\section{Introduction}

Recently, ultrahigh-channel-count and ultra-broadband fiber Bragg grating (FBG) has attracted great interests due to its excellent inter- and intra-channel performances for wavelength filtering used as the chromatic dispersion compensators [1-13] and the comb filters [10-11]. The FBGs have many advantageous features such as small size, low insertion loss, high reliability, and compatibility with other fiber components. Several kinds of methods, to date, have been proposed to design and practically fabricate the high-channel-count FBG, such as the amplitude-only sampled FBG [1-3], the super-imposed FBG [4], the amplitude-phase sampled FBG [5], the phase-only sampled FBG [6-8], the Talbot-effect based FBG [10-11], and the inverse-scattering method based FBG [12]. In particular, the phase-only sampled FBG has attracted much more interest due to the requirements of the minimum index-modulation and the smooth refractive-index profile [6-9]. High-channel count FBG with the channel up to 81, used as either the dispersion compensator or the simultaneous dispersion and dispersion slope compensator has already been demonstrated [7]. However, with further increasing the number of channels to cover the full band of wavelength-division-multiplex (WDM), optimization for the phase-only sampling function becomes extremely difficult with the present nonlinear algorithms since too large numbers of the free-parameters need to be optimally decided. It's too hard to realize a high-count-channel FBG covering the whole WDM bands by using the traditional phase-only sampling method.

Most recently, we report a continuous sampling scheme enabling one to create an ultrahigh-channel-count FBG with both high channel uniformity and high in-band energy efficiency based on two sampling functions[13]. The resulted FBG has 135 channels covering three telecom bands $(S+C+L)$. In this study, to further increase the channel number of the sampled FBG covering the whole band of fiber telecom system and meanwhile to keep the 
condition that the required maximum index-change for the designed FBG is acceptable in reality, a triple sampling method is firstly proposed and demonstrated for the design of the ultrahigh-channel-count FBG, which is based on the simultaneously utilization of two amplitude-assisted phase sampling (AAPS) functions and a phase-only sampling (POS) function. With the proposed method, more design flexibilities to the high-channel-count FBG can be obtained. As an example, one linearly chirped FBG with consecutive 1215 channels providing a wavelength range covering the whole bands $(\mathrm{O}+\mathrm{E}+\mathrm{S}+\mathrm{C}+\mathrm{L}+\mathrm{U})$ is numerically demonstrated.

\section{Principle of the triple sampling method}

In general, a sampled FBG is the product of a single-channel seed grating $\Delta n_{s g}$ with a sampling function $s(z)$. The induced refractive index-modulation $\Delta n_{s}$ can be expressed as [9]

$$
\Delta n_{s}(z)=\operatorname{Re}\left\{\Delta n_{s g}(z) \cdot s(z)\right\}=\operatorname{Re}\left\{\frac{\Delta n_{1}(z)}{2} \exp \left[i \frac{2 \pi z}{\Lambda}+i \phi_{g}(z)\right] \cdot s(z)\right\},
$$

where $\Delta n_{1}(z)$ is the maximum index-modulation, $z$ is the position along the grating, $\Lambda$ represents the average uniform period of the seeding grating, $\phi_{g}(z)$ denotes the phase-change related to the chirp properties of the seed grating. For convenience, the "dc" part of the index-modulation is neglected in Eq. (1). Whereas $s(z)$ denotes a sampling function. Unlike to the previous sampling scheme of ours [13], here the sampling function adopted is the typical one consisted of three sampling functions with relative small channel, which can be expressed as

$$
s(z)=s_{a 1}(z) \cdot s_{a 2}(z) \cdot s_{p 3}(z),
$$


where $S_{a 1}(z)$ and $S_{a 2}(z)$ both are the amplitude-assisted phase-only sampling (AAPS) function, each of them have a relative small in-band channels. Meanwhile $S_{p 3}(z)$ is a phase-only sampling (POS) function with a larger number of in-band channels. In general, these sampling functions can be expanded in Fourier series as

$$
\begin{aligned}
& S_{a 1}(z)=a_{1}(z) \cdot \exp \left[i \theta_{1}(z)\right]=\sum_{m=-\infty}^{\infty} S_{m 1} \exp \left[i 2 m \pi z / p_{1}\right] \\
& S_{a 2}(z)=a_{2}(z) \cdot \exp \left[i \theta_{2}(z)\right]=\sum_{m=-\infty}^{\infty} S_{m 2} \exp \left[i 2 m \pi z / p_{2}\right] \\
& S_{p 3}(z)=\exp \left[i \theta_{3}(z)\right]=\sum_{m=-\infty}^{\infty} S_{m 3} \exp \left[i 2 m \pi z / p_{3}\right]
\end{aligned}
$$

where $a_{1}(z)$ and $\theta_{1}(z)$ denote the amplitude and phase of the AAPS function $s_{a 1}(z)$, $a_{2}(z)$ and $\theta_{2}(z)$ denote the amplitude and phase of the AAPS function $s_{a_{2}}(z)$, $\theta_{3}(z)$ denotes the phase of the POS function $s_{p 3}(z)$. The summation terms in Eqs. (3), (4) and (5) denote the sampling functions expanded in Fourier series, respectively, where $p_{1}, p_{2}$, and $p_{3}$ are the sampling periods of the three sampling functions, and the in-band channels considered are assumed to be $2 N_{1}+1,2 N_{2}+1$, and $2 N_{3}+1$, respectively, with the condition of $N_{1} \leq N_{2}<<N_{3}$. The channel spacings in frequency domain $\Delta v_{1}, \Delta v_{2}$, and $\Delta v_{3}$ are therefore given as $\Delta v_{1}=c / 2 n_{\text {eff }} P_{1}, \Delta v_{2}=c / 2 n_{\text {eff }} P_{2}$, and $\Delta v_{3}=c / 2 n_{\text {eff }} P_{3}$, respectively. $S_{m 1}, S_{m 2}$, and $S_{m 3}$ are the complex Fourier coefficients, $n_{\text {eff }}$ is the effective index of FBG and $\mathrm{c}$ is the velocity of light in vacuum. By using the simulated annealing algorithm and Gerchberg-Saxton iterative method $[14,15]$, the sampling functions are optimized to make the absolute value of the Fourier coefficients identical within the band of interest [6,9] and the 
other Fourier coefficients beyond $|m| \leq N_{1}$ for the sampling function $s_{a 1}(z)$ and $|m| \leq N_{2}$ for the sampling function $s_{a_{2}}(z)$ are zero. Meanwhile the magnitude of $\left|\alpha_{1}(z)\right|$ and $\left|\alpha_{2}(z)\right|$ near to a unit are compulsively assumed. The figures of merit for the optimization of the Eq. (3) are given as follows:

(a) Same magnitudes of the Fourier coefficient $\left|S_{m 1}\right|$ with $m=-N_{1}, \ldots .1, \ldots \ldots, N_{1}$ for a given number of the channel $2 N_{1}+1$;

(b) $\sum_{m=-N_{1}}^{m=N_{1}}\left|S_{m 1}\right|^{2}=1$

(c) The minimum magnitude for the function of $\int_{0}^{P_{1}}\left|a_{1}(z)-1.0\right|^{2} d z$.

Once the sampling function $s_{a 1}(z)$ is optimally established, the Eq. (3) can be rewritten as

$$
S_{a 1}(z)=\sum_{m=-N_{1}}^{N_{1}} S_{m 1} \exp \left(i 2 \pi m z / P_{1}\right)
$$

For the $s_{a 2}(z)$, the optimization processes are same as those ones described above except that the in-band channel is set as $2 N_{2}+1$. So once the optimization for the sampling function $S_{a 2}(z)$ is obtained, the Eq. (4) can also be rewritten as

$$
S_{a 2}(z)=\sum_{m=-N_{2}}^{N_{2}} S_{m 2} \exp \left(i 2 \pi m z / P_{2}\right)
$$

For the phase-only sampling function $s_{p 3}(z)$ with in-band channels $2 N_{3}+1$, it can be obtained by using the same method described and utilized in Ref. [7, 9]. However, since it is phase-only one, the out-band channel cannot be eliminated.

Figure 1 shows the scheme for the triple-sampling principle based on the Fourier analysis. The detailed processes are summarized as follows: 
Step 1: As a seed grating, a single channel FBG is firstly designed by using Layer-peeling method [16].

Step 2: By utilizing the simulated annealing method and the Gerchberg-Saxton algorithm, optimization with three sampling functions are accomplished. A $\left(2 N_{1}+1\right)$ channel FBG can be generated in frequency(wavelength) domain by multiplying the AAPS function $S_{a 1}(z)$ with the seed grating in spatial domain, which could be seen in Fig. 1(b).

Step 3: In the spatial domain, the second amplitude-assisted phase sampling function $s_{a 2}(z)$ (with an in-band channel $\left.2 N_{2}+1\right)$ is then multiplied by the originated $\left(2 N_{1}+1\right)$ channel FBG (has already been obtained in the step 2), then in the frequency domain, an FBG with $\left(2 N_{1}+1\right) \cdot\left(2 N_{2}+1\right)$ channels could be obtained as is shown in Fig. 1(c). Note that, to avoid overlapping between the generated channels, the condition $P_{2} \leq P_{1} /\left(2 N_{1}+1\right)$ should be satisfied.

Step 4: The phase-only sampling function $s_{p 3}(z)$ with a higher channel-count of $2 N_{3}+1$ is then multiplied by the two amplitude-assisted phase-only sampled FBG as is shown Fig. 1(d). To avoid overlapping between the generated channels, the condition $P_{3} \leq P_{2} /\left(2 N_{2}+1\right)$ should be satisfied. Since the energy efficiency of the AAPS function is $100 \%$, the energy efficiency of the triple-sampling (AAPS $\times$ AAPS $\times$ POS) is only determined by the function $s_{p 3}(\mathrm{z})$.

Figure 1(e) shows the reflection spectrum of the realized FBG using the triple sampling method, it can be seen that one can easily obtain consecutive $\left(2 N_{1}+1\right) \cdot\left(2 N_{2}+1\right) \cdot\left(2 N_{3}+1\right)$ channels with channel spacing of $\Delta v_{1}=c / 2 n_{\text {eff }} P_{1}$ in the frequency domain as long as the conditions $p_{2}=p_{1} /\left(2 N_{1}+1\right)$ and $p_{3}=p_{2} /\left(2 N_{2}+1\right)$ are 
satisfied. Therefore, the channel number can be easily and considerably increased by using the triple sampling method. Moreover, since the out-band channel resulted from the AAPS function $s_{a 1}(z)$ and $s_{a 2}(z)$ are null, so there exist no overlap between the channels.

Note that, by choosing the suitable magnitudes for the sampling period, some special multichannel FBGs having either non-consecutive channels or step-changeable channel-spacing could be obtained under the condition of $p_{2}<p_{1} /\left(2 N_{1}+1\right)$ and $p_{3}<p_{2} /\left(2 N_{2}+1\right)$, which in return means that more design flexibilities for the high-channel-count FBG could be obtained with the proposed sampling approach.

\section{Design results utilizing the triple double sampling method}

To verify the above proposals, design for a linearly chirped FBG with 1215 channels is implemented. Firstly, two AAPS functions with 3-, 9-channel and a POS function with 45-channel are optimal designed, which are shown in Figs. 2, 3, and 4, respectively. Figures 2(a) and 2(b) show the amplitude and phase distribution of the 3-channel sampling function normalized in one period. Figure 2(c) shows the obtained channel spectrum in which the in-band energy (diffraction) efficiency is 100\%. Figures 3(a) and 3(b) show the amplitude and phase distribution of the 9-channel sampling function normalized in one period. It can be seen that there exists a quasi-periodical and low-frequency oscillation on the amplitude of the 9-channel sampling function. Figure 3(c) shows the obtained channel spectrum in which the in-band energy (diffraction) efficiency is also 100\%. Figure 4(a) shows the phase distribution of the 45-channel phase-only sampled function normalized in one period. Figure 4(b) shows the obtained channel spectrum in which the non-uniformity over all 45 channels is less than $0.5 \%$, and the in-band energy (diffraction) efficiency is larger than 93\%. The sampling period of the 3-channel sampling functions $P_{1}$ is adopted as $2 \mathrm{~mm}$ (i.e., the channel spacing is $50 \mathrm{GHz}$ ). To 
satisfy the condition $P_{2}=P_{1} /\left(2 N_{1}+1\right)$ and $P_{3}=P_{2} /\left(2 N_{2}+1\right)$, the sampling periods of the 9and 45-channel sampling functions are given as 2.0/3 and 2.0/27 mm, respectively. Secondly, a seed grating is designed by using the layer peeling method [16]. The seed grating is designed to have the chromatic dispersion compensation of $-1360 \mathrm{ps} / \mathrm{nm}$, a length of $9 \mathrm{~cm}$, and the $0.5 \mathrm{~dB}$ bandwidth of $37.5 \mathrm{GHz}$. The design results are illustrated in Fig. 5, where Fig. 5(a) shows the index-modulation and phase profile of the seed grating, Fig. 5(b) shows its calculated reflection and group delay spectra.

By multiplying the seed grating with the 3-, 9- and 45-channel sampling functions simultaneously in spatial domain, an ultrahigh channel-count FBG with 45 sets of $3 \times 9$-channel (i.e., 1215 consecutive channels) is realized. The design results of the 1215-channel triple sampled FBG (AAPS+AAPS+POS) are illustrated in Fig. 6. Figure 6(a) shows the index modulation, the inset shows the fine index profile within $2.0 \mathrm{~mm}$ region in the grating direction. It can be seen that the maximum index-modulation is about $6 \times 10^{-3}$, which is obtainable in practice with the heavily hydrogen-loaded photosensitive fiber. There also exists a cosine-like oscillation on the grating profile and period of this oscillation is about $2.0 / 27 \mathrm{~mm}$, which is determined by the two (3- and 9-channel) AAPS functions as shown in Fig. 2(a) and Fig. 3(b), respectively. In concrete, period of this oscillation is equal to a specific magnitude, i. e., the sampling period divided by the channel number. In our case, we choose the sampling period of $2 \mathrm{~mm}$ (corresponding to $50 \mathrm{GHz}$ channel spacing), one POS with channel of 45 (which will result in no oscillation on the index-modulation profile), and two AAPS with channel 3 and 9, respectively, then period of the resulted oscillation will be equal to $2 \mathrm{~mm} /(3 \times 9) \approx 74 \mu \mathrm{m}$ which agrees well with the one shown in the inset of Fig. 6(a). Note that since the size of the UV writing beam should be at least two times less than the period of the oscillation according to the Nyquist sampling theorem, we expect that it will be much more 
difficult to fabricate this kind of high channel-count FBG than the single-channel one. However, by using the advanced phase-mask writing technique incorporated with a narrow UV beam (with beam size less than $10 \mu \mathrm{m}$ ) [17], it is possible for one to write this kind of grating with a high quality.

The calculated reflection and group delay spectra of the 1215-channel FBG covering a frequency range of $60.75 \mathrm{THz}$ are shown in Fig. 6(c). To illustrate the spectra clearly, the three channels locating at the frequencies of $187.05 \mathrm{THz}, 193.05 \mathrm{THz}$, and $199.05 \mathrm{THz}$ are given in the insets of Fig. 6(c). Moreover, the group delay ripples for all in-band channels are smaller than \pm 2 ps.

\section{Conclusion}

A triple sampling method enabling to have an excellent channel uniformity and high in-band energy efficiency is proposed for the design of an ultrahigh-channel-count FBG. Based on the proposed method, a linearly chirped FBG having a length of $9 \mathrm{~cm}$, a dispersion of $-1360 \mathrm{ps} / \mathrm{nm}$, and a channel spacing of $50 \mathrm{GHz}$, and with consecutive channels of 1215 is numerically demonstrated. The maximum index-change required for $10 \mathrm{~dB}$ strength of the FBG is about $6 \times 10^{-3}$. There exist no distortions on the reflection and group delay spectra of the ultrahigh-channel-count FBG. The proposed scheme may be extended to the design of the specially sampled FBGs with either non-consecutive channels or non-identical channel-spacing ones.

\section{Acknowledgments}

This work was partly supported by the Grant-in-Aid for Scientific Research from the Japan Society for the Promotion of Science. 


\section{References}

1. F. Ouellette, P. A.Krug, T. Stephens, G. Dhosi and B. Eggleton, "Broadband and WDM dispersion compensation using chirped sampled fibre Bragg gratings,” Elec. Lett. 31, 899-901 (1995).

2. W. H. Loh, F. Q. Zhou, and J. J. Pan, "Sampled fiber grating based -dispersion slope compensator," IEEE Photon. Tech. Lett. 11, 1280-1282 (1999).

3. M. Ibsen, M. Durkin, M. Cole and R. Laming, "Sinc-sampled fiber Bragg gratings for identical multiple wavelength operation,” IEEE Photon. Tech. Lett. 10, 842-844 (1998).

4. Y. Painchaud, A. Mailloux, H. Chotard, E. Pelletier, and M. Guy, "Multi-channel fiber Bragg gratings for dispersion and slope compensation,” Optical Fiber Communication Conf. Paper. ThAA5. (2002).

5. A. V. Buryak K. Y. Kolossovski, and D. Y. Stepanov, "Optimization of refractive index sampling for multichannel fiber Bragg gratings,” IEEE J. Quantum Electron., 39, 91-98(2003).

6. J. E. Rothenberg, H. Li, Y. Li, J. Popelek, Y. Wang, R. B. Wilcox, and J. Zweiback, “Dammann fiber Bragg gratings and phase-only sampling for high channel counts,” IEEE Photon. Technol. Lett., 14, 1309-1311 (2002).

7. H. Li, Y. Sheng, Y. Li, J. E. Rothenberg, "Phased-only sampled fiber Bragg gratings for high channel counts chromatic dispersion compensation,” IEEE/OSA J. Lightwave Technol. 21, 2074-2083 (2003).

8. H. Lee and G. Agrawal, "Bandwidth equalization of purely phase-sampled fiber Bragg gratings for broadband dispersion and dispersion slope compensation,” Opt. Express, 12, 5595-5602 (2004). 
9. H. Li, M. Li, Y. Sheng, and J. E. Rothenberg, “Advances in the design and fabrication of high-channel-count fiber Bragg gratings,” J. Lightwave Technol. 25, 2739-2750 (2007).

10. J. Magne, P. Giaccari, S. LaRochelle, J. Azana, L. R. Chen, “All-fiber comb filter with tunable free spectral range,” Opt. Lett. 30, 2062-2064 (2005).

11. C. Wang, J. Azana, L. R. Chen, "Spectral Talbot-like phenomena in one-dimensional photonic bandgap structures,” Opt. Lett. 29, 1590-1592 (2004).

12. H. Li and Y. Sheng, "Direct design of multi-channel fiber Bragg grating with discrete layer-peeling algorithm,” IEEE Photon. Technol. Lett., 15, 1252-1254 (2003).

13. M. Li, X. Chen, J. Hayashi, and H. Li, “Advanced design of the ultrahigh-channel-count fiber Bragg grating based on the double sampling method,” Opt. Express 17, 8382-8394 (2009).

14. R. W. Gerchberg and W. O. Saxton, “A practical algorithm for the determination of phase from image and diffraction image and diffraction plane pictures,” Optik, 35, 237-246(1972).

15. S. Kirkpatrick, C. D. Gelatt, Jr. and M. P. Vecchi, “Optimization by simulated annealing,” Science, 220, 671-680(1983).

16. J. Skaar, L. Wang, and T. Erdogan, “On the synthesis of fiber Bragg grating by layer peeling,” IEEE J. Quantum Electron., 37, 165-173(2001).

17. Y. Painchaud and M. Morin, "Iterative method for the design of arbitrary multi-channel fiber Bragg gratings,” in OSA Topical Meeting Bragg Gratings, Photosensitivity, and Poling in Glass Waveguides (BGPP2007), paper BTuB1. 


\section{List of figure captions:}

Fig. 1. Fourier analysis of the proposed triple sampling method for the design of ultrahigh-channel-count, ultra-broadband FBG.

Fig. 2. Design results for a 3-channel AAPS function. (a) amplitude profile, (b) phase profile, and (c) channel spectrum.

Fig. 3. Design results for a 9-channel AAPS function. (a) Amplitude profile, (b) Phase profile, and (c) channel spectrum.

Fig. 4. Design results for a 45-channel phase-only sampling function. (a) Phase profile, and (b) channel spectrum.

Fig. 5. Design results for a one-channel FBG based on the inverse scatter method. (a) Grating index- and phase- profile, and (b) the reflection and group delay spectra.

Fig. 6. Design results for a 1215-channel triply sampled FBG (AAPS $\times$ AAPS $\times$ POS). (a) Grating index profile, the inset shows the fine index profile in a region of $2 \mathrm{~mm}$, (b) grating phase profile, the inset shows the fine phase profile in a distance of $1 \mathrm{~mm}$, (c) reflection and group delay spectra, the insets show the spectra at the central frequency of $187.05 \mathrm{THz}, 193.05$ $\mathrm{THz}$, and $199.05 \mathrm{THz}$, respectively. 


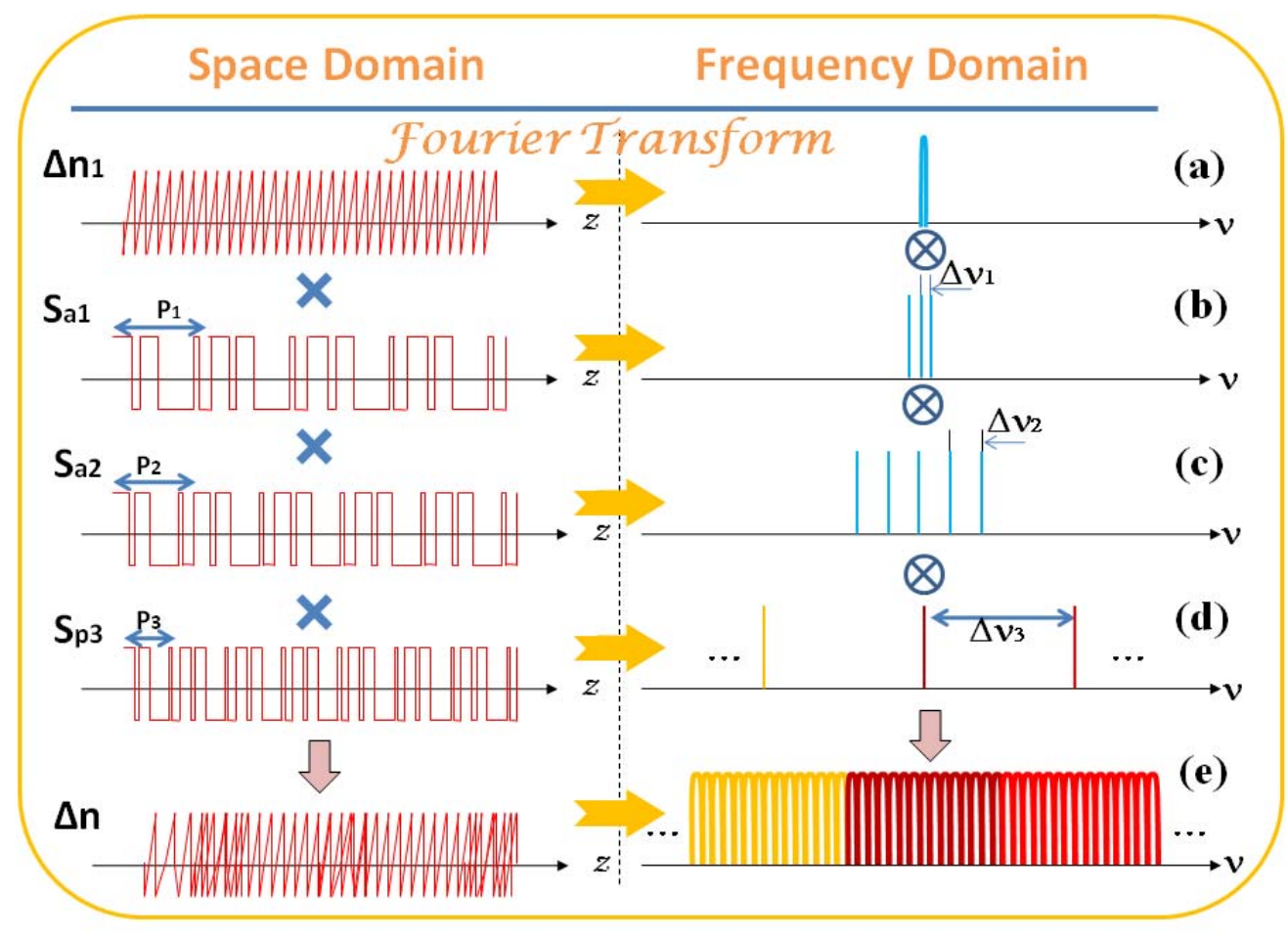

Fig. 1. Fourier analysis of the proposed triple sampling method for the design of ultrahigh-channel-count, ultra-broadband FBG. 

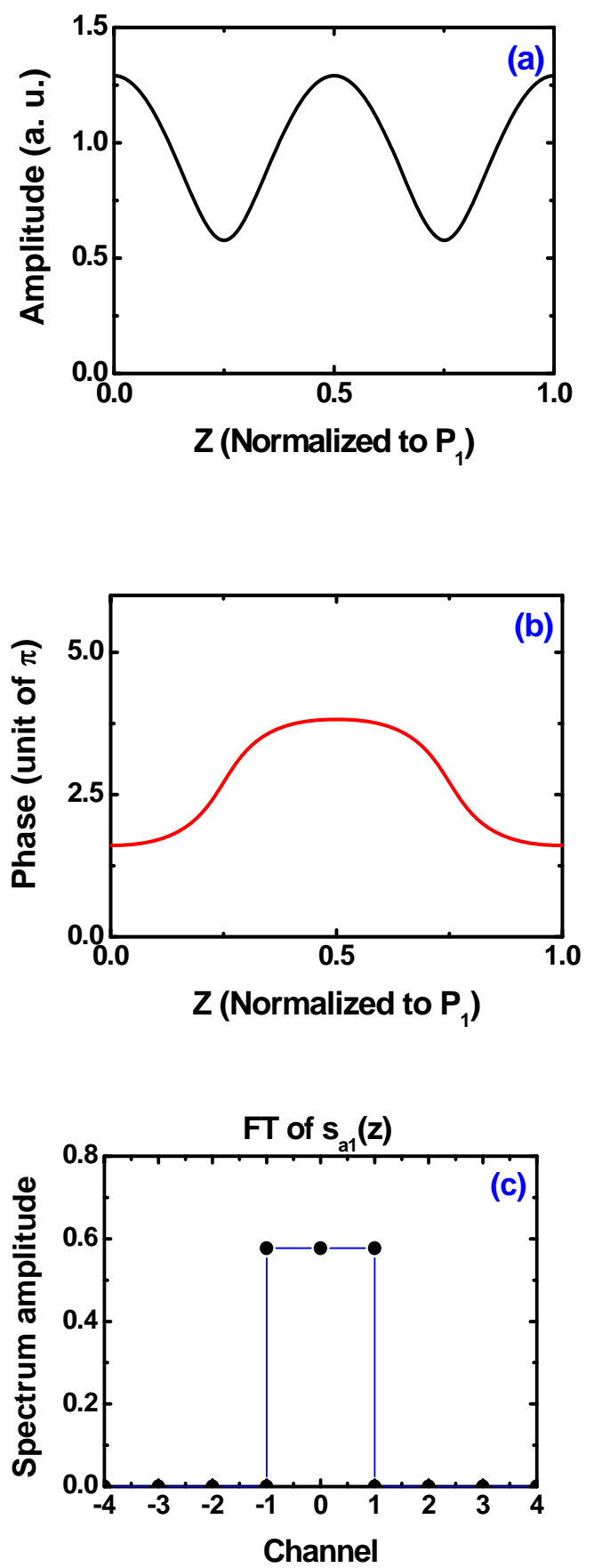

Fig. 2. Design results for a 3-channel AAPS function. (a) amplitude profile, (b) phase profile, and (c) channel spectrum. 

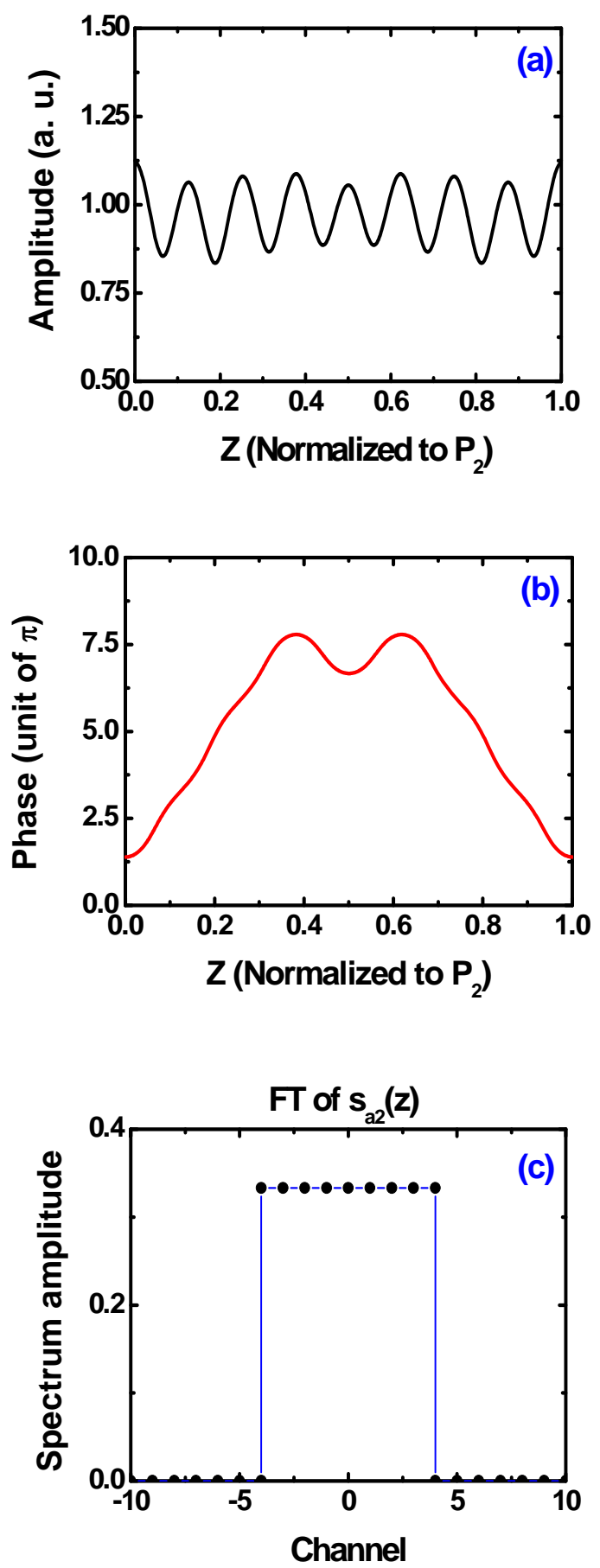

Fig. 3. Design results for a 9-channel AAPS function. (a) Amplitude profile, (b) Phase profile, and (c) channel spectrum. 

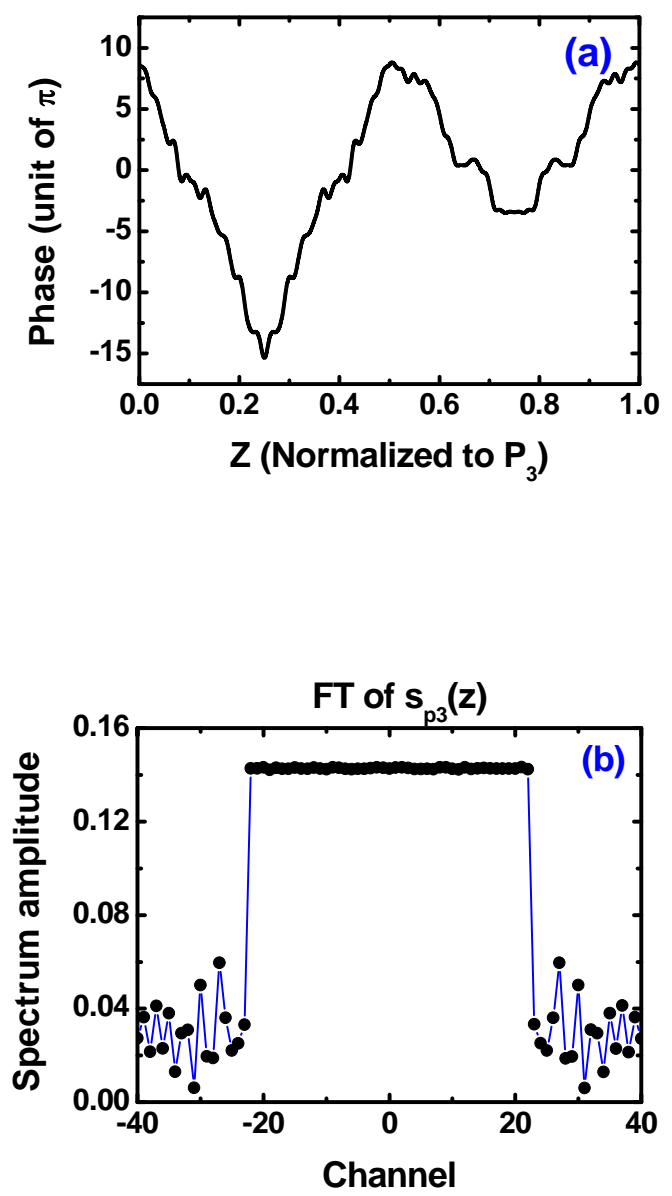

Fig. 4. Design results for a 45-channel phase-only sampling function. (a) Phase profile, and (b) channel spectrum. 

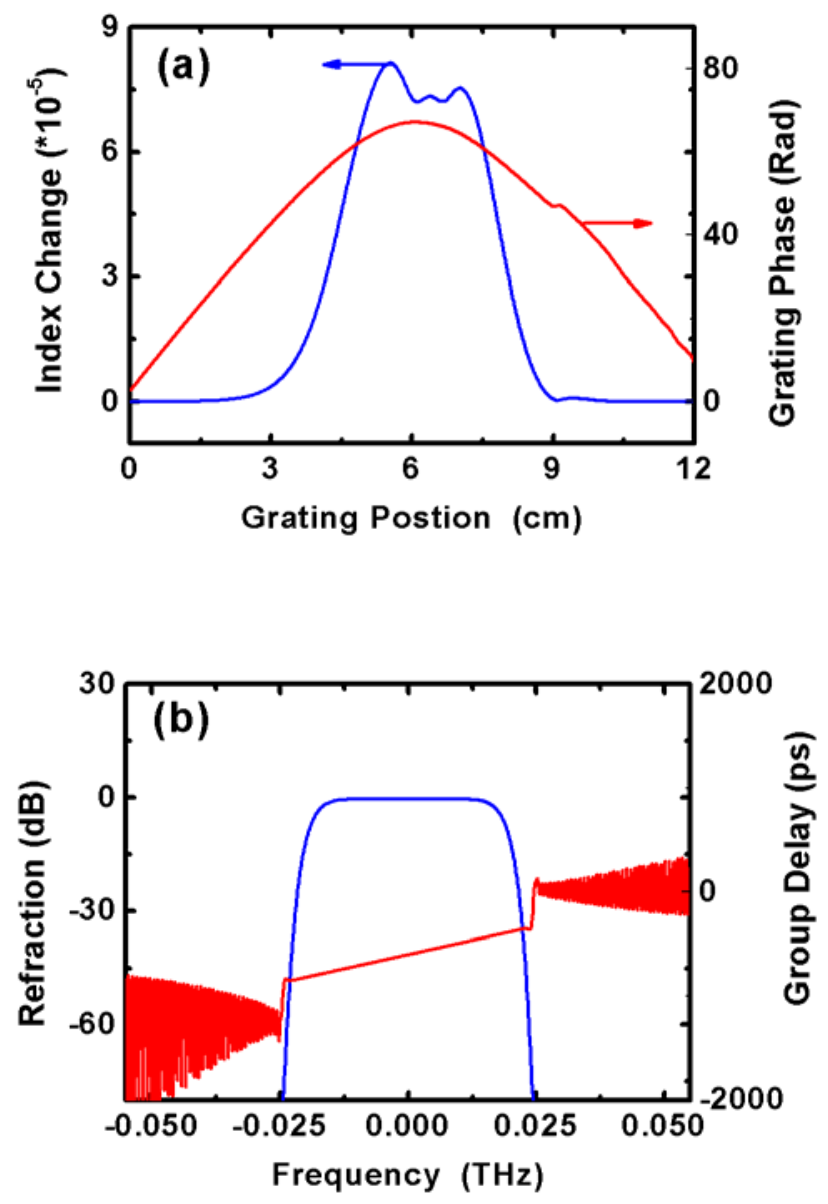

Fig. 5. Design results for a one-channel FBG based on the inverse scatter method. (a) Grating index- and phase- profile, and (b) the reflection and group delay spectra. 

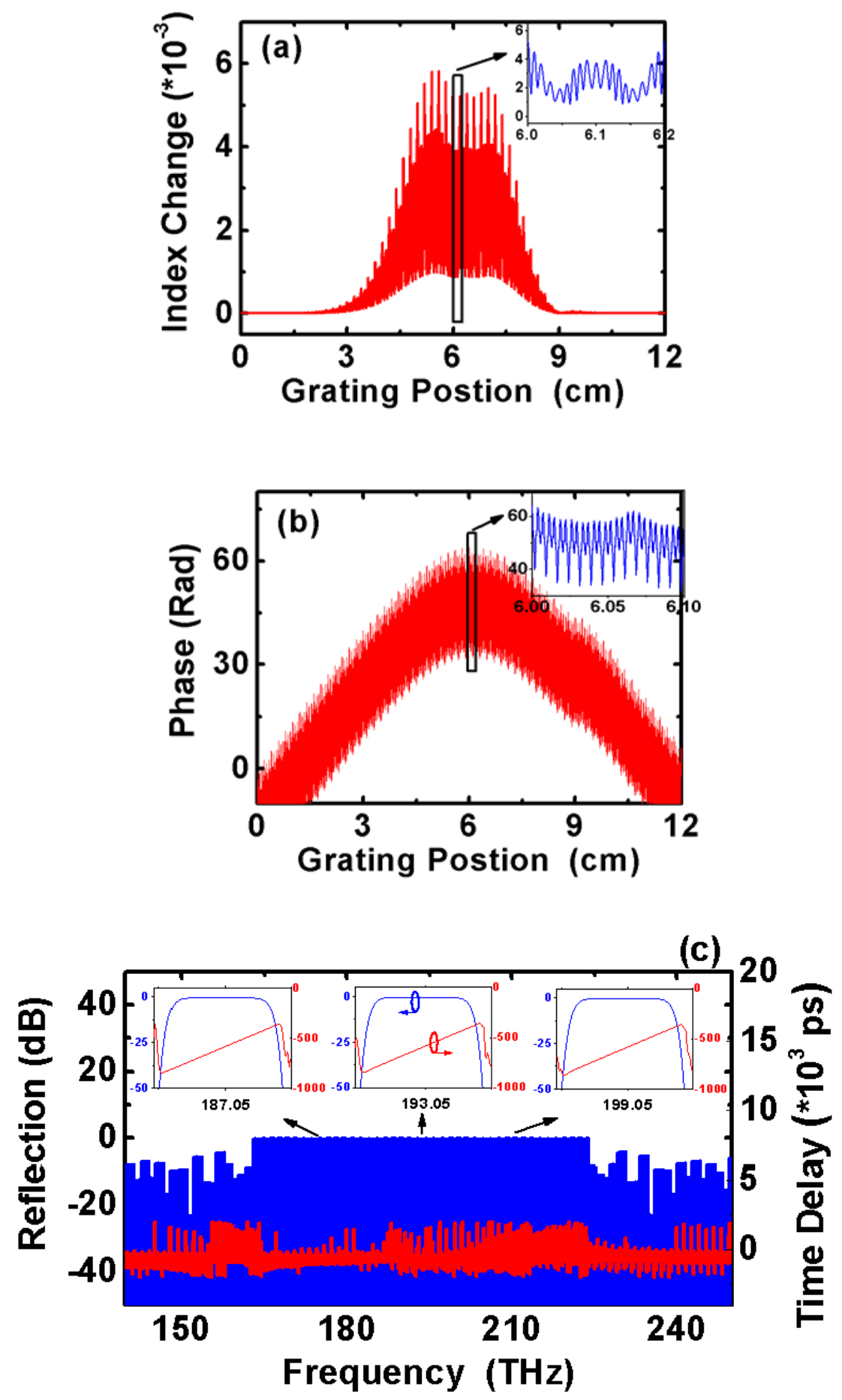

Fig. 6. Design results for a 1215-channel triply sampled FBG (AAPS $\times$ AAPS $\times$ POS). (a) Grating index profile, the inset shows the fine index profile in a region of $2 \mathrm{~mm}$, (b) grating phase profile, the inset shows the fine phase profile in a distance of $1 \mathrm{~mm}$, (c) reflection and group delay spectra, the insets show the spectra at the central frequency of $187.05 \mathrm{THz}, 193.05 \mathrm{THz}$, and $199.05 \mathrm{THz}$, respectively. 